\title{
Investigating information-seeking behaviors of primary care physicians who care for older depressed patients and their family caregivers: a pilot study
}

\author{
Mary Jo Dorsey and Ellen Detlefsen
}

\begin{abstract}
Objective - To describe preliminary findings from a study of information-seeking behaviors of primary care physicians who care for elderly and depressed patients, and the correlation between what is sought versus what is provided to the patient and (or) caregiver. Setting - Physicians in two large ambulatory primary care practices throughout urban Pittsburgh, Pennsylvania, who take care of geriatric patients. Methods - Structured interviews, with common questions, will be conducted with 12 primary care physicians to determine patterns of information-seeking behaviors. Environmental scans of physicians' offices for evidence of their existing information behaviors will complement the information obtained from the interviews. Results - This pilot study provides an analysis of the resources primary care physicians use to seek information to provide to patients and caregivers. Analyses show types of information sought, time spent seeking information, and methods used to find information given to patients. Conclusions - With mounting evidence of the Internet being used for patient self care, it is essential to understand if primary care physicians understand the scope and breadth of information readily available to their patients. The primary care physician needs to be aware of the types of information made available to their patients and the caregivers who are inclined to obtain information for the patient.
\end{abstract}

\section{Introduction}

Over the past decade, studies on the information-seeking behavior of physicians have highlighted the paths physicians use to obtain clinical literature for the purposes of practicing evidence-based medicine. This study examines the methods physicians use to obtain consumer information for patients and their caregivers. Specifically shown are findings from a pilot study of the information-seeking behaviors of primary care physicians who care for elderly and depressed patients and the correlation between what is sought versus what is provided to the patient and (or) caregiver.

For the purpose of this study, the term "information-seeking behavior" refers to a physician's methods of and attitudes toward the process of locating relevant information for clinical or patient education uses. Physicians' behaviors include the following: (1) the use of printed information from textbooks, journals, pamphlets, and pharmacological product inserts in hard copy; (2) the use of electronic or digital media, includ- ing online or electronic textbooks, databases, journals and Web sites, and audiovisual materials, either personally owned or library owned; and (3) conversing with colleagues or others knowledgeable in a field of expertise via the telephone, in person, or through some electronic mechanism. Vehicles used to gather information include paper, computers, electronic media, and personal correspondence via telephone, fax, computer (e-mail, chat), or face-to-face conversations. In 2000, McKnight and Peet's study of health care providers' information seeking [1] found that in spite of the onset of the availability of online resources, clinicians were still using older, more traditional ways of gathering information.

Although looking for information in an electronic atmosphere is becoming ubiquitous, textbooks and personal contacts remain significant. Bennett et al. [2] indicated that approximately half of the physicians polled in a 2001 survey noted that the Internet made a significant impact on the way they conducted their practices. Conversely, in 2004, Bryant [3] cited that four-fifths of the interviewees declared that refer-

M.J. Dorsey. ${ }^{\mathbf{1}}$ Richard M. Johnston Health Sciences Library, The Western Pennsylvania Hospital, 4800 Friendship Avenue,

Pittsburgh, PA 15224, USA, and the Department of Library \& Information Science, School of Information Sciences, University of Pittsburgh, 135 N. Bellefield Avenue, Pittsburgh, PA 15260, USA.

E. Detlefsen. Department of Library \& Information Science, School of Information Sciences; Center for Biomedical Informatics, School of Medicine and the NIMH Advanced Center in Interventions and Services Research for Late Life Mood Disorders, School of Medicine, University of Pittsburgh, 135 N. Bellefield Avenue, Pittsburgh PA 15260, USA.

${ }^{1}$ Corresponding author (e-mail: mjd21@pitt.edu). 
Fig. 1. Time spent information seeking. CME, continuing medical education; EBM, evidence-based medicine.

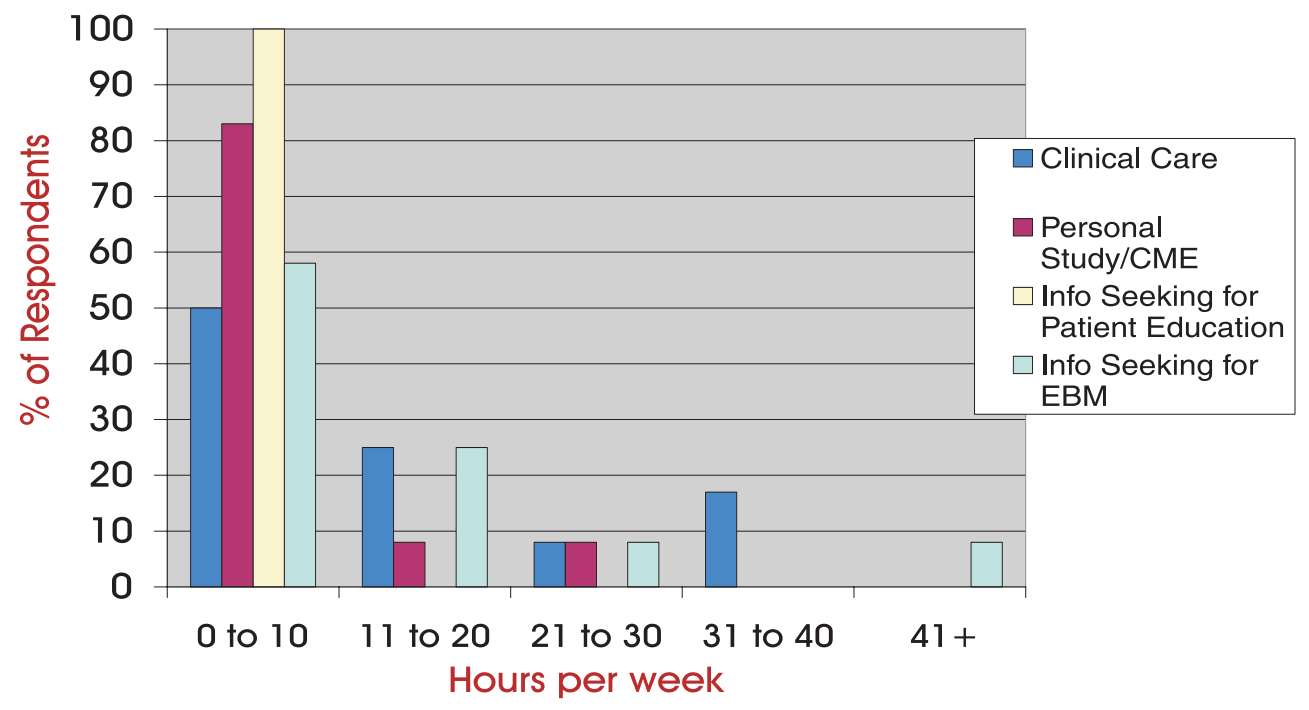

ence to printed information within the practice (to verify reference data and to aid diagnosis) was the most common information-seeking behavior. Ranking second was the use of electronic information sources. Physicians in this survey did not feel comfortable with the levels of their searching skills and implied that doing their searches in conjunction with an information professional would yield the best results.

Casebeer et al. [4] point out in a 2002 study that $80 \%$ of the respondents used the Internet on a consistent basis to retrieve information. Access to self-growth factors such as continuing medical education (CME), rapid self-performed literature searches, and access to solutions to patients' problems combined with immediacy, accuracy, and ease of use contributes not only to further knowledge, but to improved patient care as well. D'Alessandro et al.'s 2004 study [5] reports a dwindling of paper resource usage with a noteworthy rise in electronic resource use. Nylenna and Aasland [6] studied the effects of keeping up with medical literature in the information explosion era. Although the study reflected the trends of Norwegian doctors specifically, it notes a unique thought: with the flood of information needed to stay current in today's medical world, the onset of the Internet may be presenting more of a gap between doctors who seek new professional knowledge and those who do not. Those who have access and are on board with electronic information seeking are fully on board. Those who have not met that reality yet are falling behind on the learning curve. This gives information professionals a distinct purpose in the future of consumer health information proliferation. How can these analyses be used to prepare physicians for the advancement of evidencebased consumer health information?

\section{Setting and methods}

Physicians in two large primary care practices in urban Pittsburgh, Pennsylvania, who care for geriatric patients, were asked to complete a survey using SurveyMonkey ${ }^{\circledR}$ software [7]. The survey, containing 10 common questions, was administered to 12 primary care physicians to determine pat- terns of their information-seeking behaviors. Screen shots of the survey appear in Appendix A.

\section{Results}

\section{Demographics}

One hundred percent of the respondents were licensed medical doctors with the M.D. degree. The majority (91\%) were board certified in internal medicine, while the remaining participants had certifications in geriatrics, family practice, and one certification from the Royal College of Physicians. Slightly more than one-half of the participants have been in practice for more than 10 years, while less than one-half have been in practice for 0-10 years.

\section{Question 1 - practice of depressed elderly}

Physicians reported that from $5 \%$ to $30 \%$ of their work week consisted of treating elderly patients.

\section{Question 2 - time spent information seeking}

Figure 1 shows the time spent information seeking in hours per week. The types of information sought were divided into four categories, and the time spent searching each was evaluated by the participants. The categories were the following: information for clinical care; personal study or CME; information for patient education; and information for evidencebased practice methods. Overall, $0-10 \mathrm{~h}$ per week was the average answer calculated for all categories of time spent seeking information.

Question 3 - electronic devices used in office of practice

Confirming the ease of access to electronic resources, $100 \%$ reported using a computer; 92\% had Internet access in their clinical office setting; and 50\% used a PDA in the clinical setting.

\section{Question 4 - most common methods used to find} information to give to patients

Participants were asked to list the percentage of time used on each of the 14 methods of gathering information. Of the 
Fig. 2. Most common methods used to find information for patients.

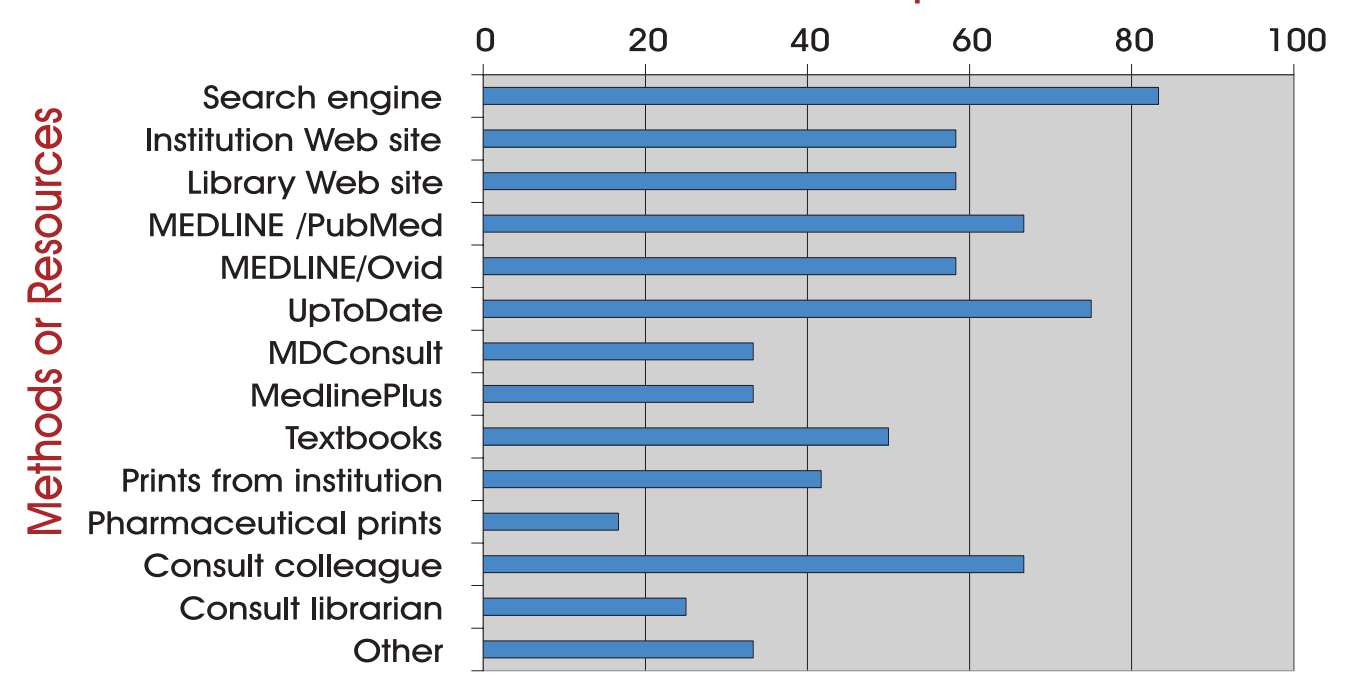

Fig. 3. Information on specific topics.

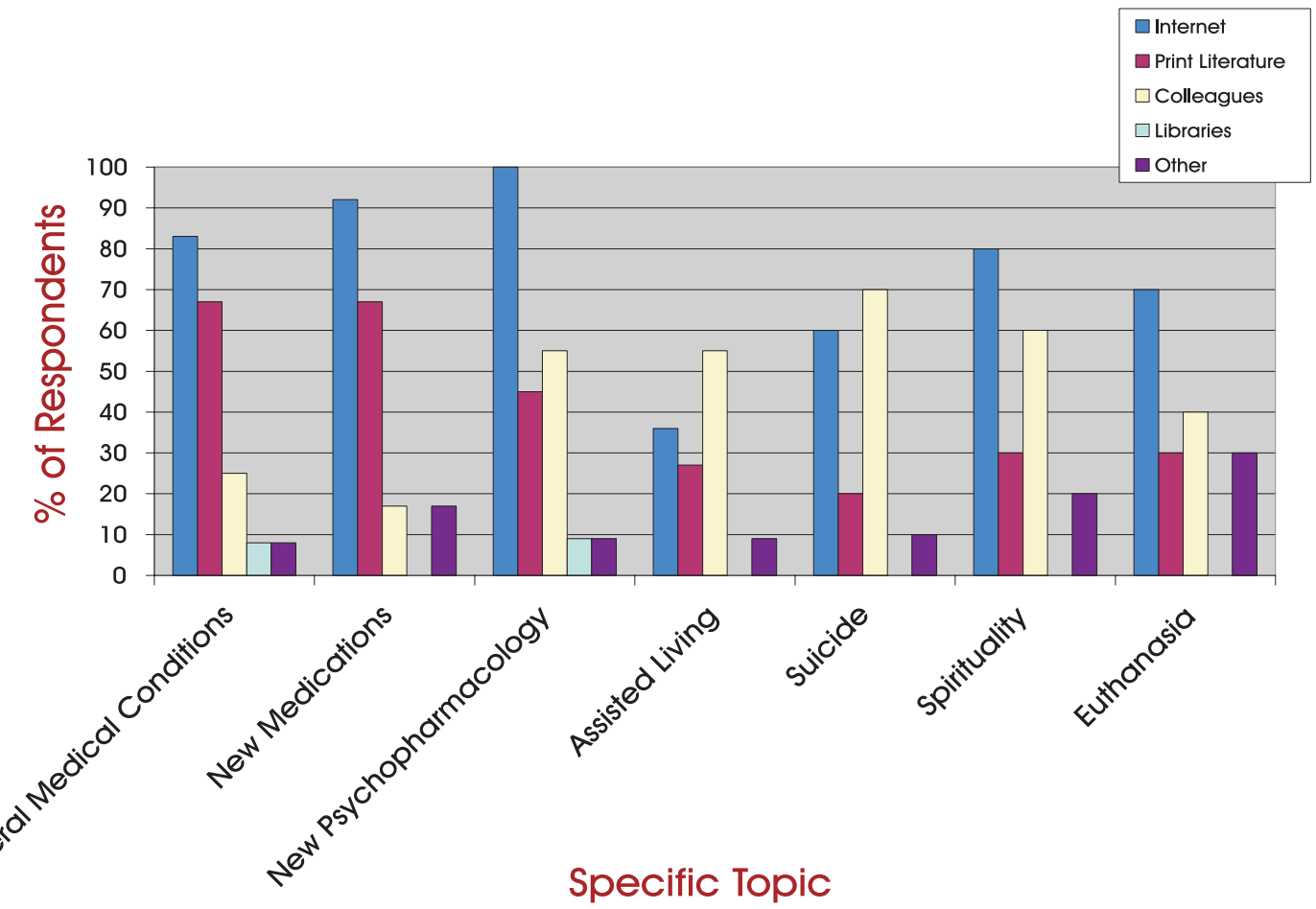

14 options listed, each was selected at least once. However, the top three methods of information seeking used to give information to patients were the following: (1) use of an Internet search engine; (2) use of a specific software product, such as UpToDate (UpToDate, Waltham, Mass.); and (3) use of a colleague's advice obtained via electronic mail, telephone, or in person. Figure 2 shows the range of results.

\section{Questions 5 and 6 - questions on specific topics}

The physicians were then asked where they tend to go for information in relation to specific topics. Participants were asked to consider whether they went to the Internet, printed literature, colleagues, a library, or to another place when they were seeking information in the following categories: general medical conditions, new medications, new psychopharmacology, assisted living, suicide, spirituality, and euthanasia. While the Internet (and to a lesser degree, print materials) remained a strong source of reference for medical conditions and pharmacology, consultation with colleagues along with the use of the Internet were the clear choices when it came to obtaining information on more elderly specific topics such as assisted living, suicide, spirituality, and euthanasia resources (Fig. 3). The category "other" included use of UpToDate, area adult services, area agencies on aging, chaplains, religious texts, and the software product Epocrates ${ }^{\circledR}$ (Epocrates, Inc., San Mateo, Calif.). Interestingly, a library 
Fig. 4. Resources located within physician's clinical office setting.

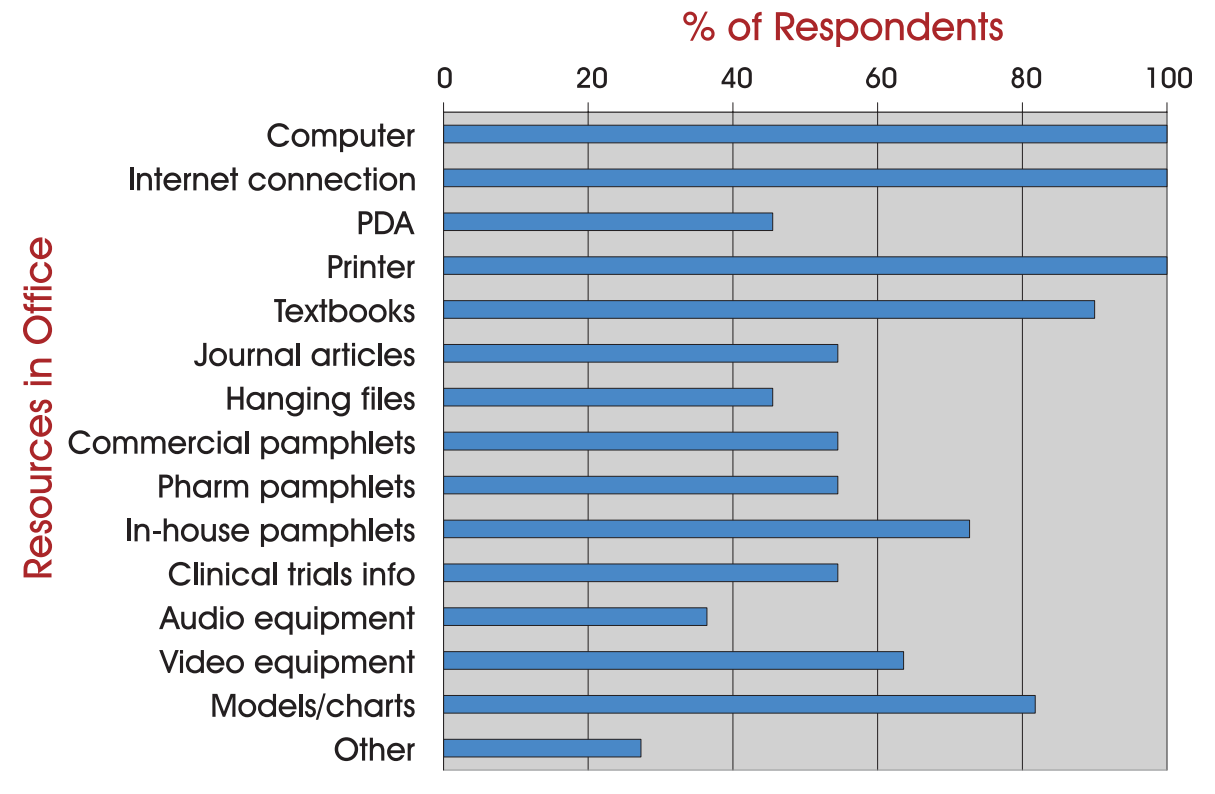

was consulted less than $10 \%$ of the time consistently across the spectrum of these specific topics.

\section{Question 7 - office scan}

In lieu of actually visiting the physician's office to perform a visual scan, this question asked the participant to report on the information resources and tools in their personal, clinical office setting. Of the 14 objects listed for identification, all respondents reported having a computer with a connection to the Internet along with a printer. Textbooks, models, and charts rounded out the remaining popular information venues within the office. Institutional (in-house) pamphlets and video equipment were somewhat popular and located within the office settings. Commercially produced pamphlets by pharmaceutical or medical device companies, PDAs, hanging files (of copied articles), clinical trial information, and audio information were among the less frequently consulted information resources for patient education. Full results are shown in Fig. 4.

\section{Discussion}

With mounting evidence of the Internet being used for patient self-care, it is essential to understand if primary care physicians understand the scope and breadth of information readily available to their patients. In the instance of the elderly, particularly the depressed elderly, the primary care physician needs to be aware of the types of information made available to their patients, including the caregivers who are inclined to obtain information for the patient.

In this small sampling of physicians, the most common methods of gathering information for patient education appear to be the following: ( $i$ ) using an Internet search engine, (ii) using professional resources such as UpToDate, and (iii) consulting a colleague. The least common methods of gathering information for patient education appear to be the following: ( $i$ ) using preprinted materials from pharmaceutical or medical device companies, (ii) consulting a librarian, and (iii) searching specific authoritative resources such as MDConsult or MedlinePlus.

Please note that this study is the preliminary work for a larger, multimodal study to be undertaken in 2005, looking at primary care physicians who are participating in a research network affiliated with a newly funded center for interventions and services research on late-life mood disorders. This was a test of a novel methodology and data collection method. One reason for the study was to see if these physicians would be willing to answer a Web-delivered questionnaire instead of a paper and pencil or telephone survey. Another reason for the study was to see if the categories of resources and tools were likely candidates for a larger survey to be conducted with added environmental scans.

\section{References}

1. McKnight M, Peet M. Health care providers' information seeking: recent research. Med Ref Serv Q. 2000 Summer;19(2):2750 .

2. Bennett NL, Casebeer LL, Kristofco RE, Strasser SM. Physicians' Internet information-seeking behaviors. J Contin Educ Health Prof. 2004 Winter;24(1):31-8.

3. Bryant SL. The information needs and information seeking behaviour of family doctors.

Health Info Libr J. 2004 Jun;21(2):84-93.

4. Casebeer L, Bennett N, Kristofco R, Carillo A, Centor R. Physician Internet medical information seeking and on-line continuing education use patterns. J Contin Educ Health Prof. 2002 Winter;22(1):33-42.

5. D'Alessandro DM, Kreiter CD, Peterson MW. An evaluation of information-seeking behaviors of general pediatricians. $\mathrm{Pe}$ diatrics. 2004 Jan;113(1 Pt 1):64-9.

6. Nylenna M, Aasland OG. Primary care physicians and their information-seeking behaviour. Scand J Prim Health Care. 2000 Mar;18(1):9-13.

7. SurveyMonkey.com. SurveyMonkey.com [computer program]. Portland, Oreg.: SurveyMonkey.com; 2004 [cited 2005 Apr 6]. Available from http://surveymonkey.com/home.asp. 


\section{Appendix A}

Fig. A1 (a). Screen shot of survey.

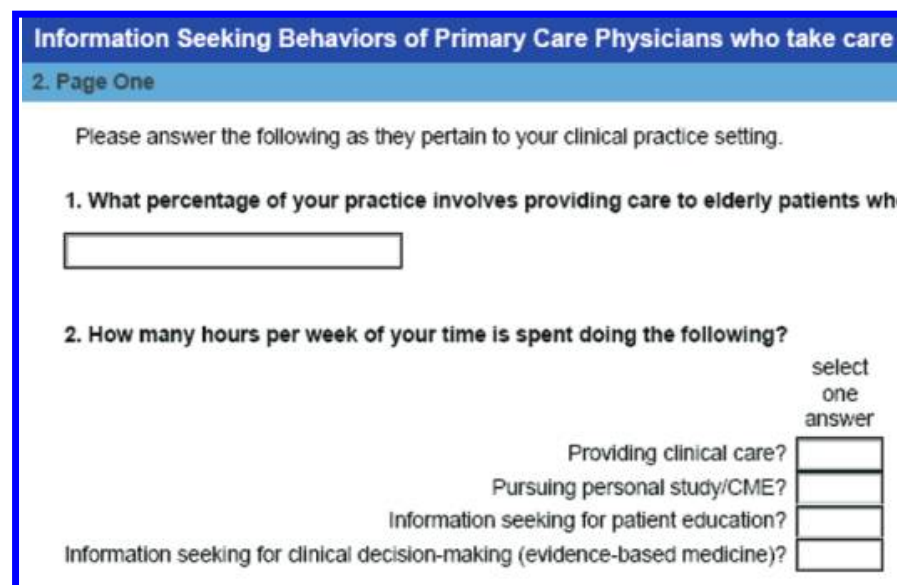

3. Place a check in the box next to items that you yourself use in your office.

$\Gamma$ computer

$\Gamma$ Internet access

$\Gamma \mathrm{PDA}$

4. Place a check in the box next to each of the methods you use to find information that you give to your patients. You may check more than one.

$\Gamma$ Search Internet using a search engine such as Google

Search Internet using your institution's website and resources

$\Gamma$ search a Library website

$\Gamma$ Search professional resources such as MEDLINE/PubMed

$\Gamma$ search professional resources such as MEDLINE/Ovid

$\Gamma$ Search professional resources such as UPTODate

$\Gamma$ search professional resources such as MDConsult

$\Gamma$ search consumer health websites such as MedlinePlus

$\Gamma$ scan textbooks or printed materials from a personal collection

$\Gamma$ Offer pre-printed materials from your institution

$\Gamma$ Offer pre-printed materials from a pharmaceutical or medical device agency

$\Gamma$ consult a colleague

$\Gamma$ consult a Librarian

$\Gamma$ other (please specify)

5. When you need information on these specific topics for a patient or family member, where do you typically go?

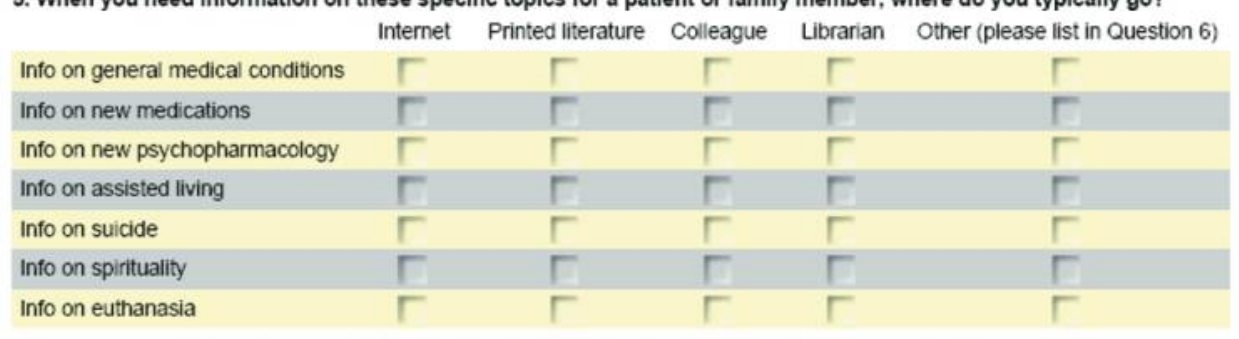

6. If you entered "Other" to any of the selections in Question 5, please list where you go for information.

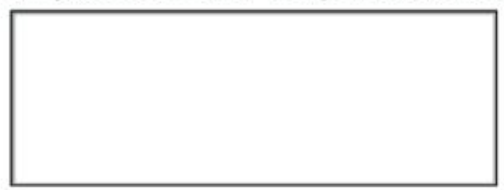


Fig. A1 (b).

Information Seeking Behaviors of Primary Care Physicians who take care of older depressed patients

2. Page One

Please answer the following as they pertain to your clinical practice setting.

1. What percentage of your practice involves providing care to elderly patients who may be depressed?

2. How many hours per week of your time is spent doing the following?

\section{select}

one

answer

Providing clinical care?

Pursuing personal study/CME?

Information seeking for patient education?

Information seeking for clinical decision-making (evidence-based medicine)?

3. Place a check in the box next to items that you yourself use in your office.

Computer

Internet access

TPDA

4. Place a check in the box next to each of the methods you use to find information that you give to your patients. You may chech more than one.

Tearch Internet using a search engine such as Google

Tearch Internet using your institution's website and resources

T Search a Library website

Tearch professional resources such as MEDLINE/PubMed

- Search professional resources such as MEDLINE/Ovid

Tearch professional resources such as UpToDate

T Search professional resources such as MDConsult

Tearch consumer health websites such as MedlinePlus

T scan textbooks or printed materials from a personal collection

Offer pre-printed materials from your institution

Offer pre-printed materials from a pharmaceutical or medical device agency

T Consult a colleague

T Consult a Librarian

Tother (please specify)

5. When you need information on these specific topics for a patient or family member, where do you typically go? Internet Printed literature Colleague Librarian Other (please list in Question 6)

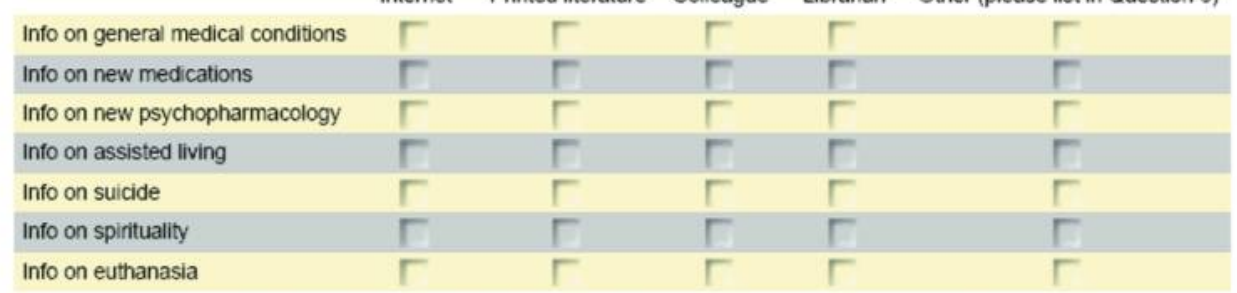

6. If you entered "Other" to any of the selections in Question 5, please list where you go for information. 